Children as Caregivers: The Global Fight Against Tuberculosis and HIV in Zambia, by Jean Hunleth. Rutgers, NJ: Rutgers University, 2017.

In Children as Caregivers: The Global Fight Against Tuberculosis and HIV in Zambia, anthropologist and public health researcher Jean Hunleth explores "how intergenerational care happens when infectious disease becomes woven into the structure, relationships, and rhythms of day-of-day life" (p. 3). Working in George Compound in Lusaka, Zambia, Hunleth centers her ethnographic research of "the continued pursuit of universal treatment for TB and HIV" (p. 3) on the experiences and voices of children with ill family members. In so doing, she addresses the broad erasure of children from both public health research, and studies of caregiving and care work. She eloquently demonstrates the very real role and work that children do in caring for their ill parents and supporting broader kin-networks. The affective relationships among children and adults in George are central to the treatment of TB and HIV. Through her experience researching both infectious disease and children, Hunleth offers engaged scholars important lessons about the agency of children within intergenerational caregiving and including children as expert participants.

Hunleth's multidisciplinary research experience with the delivery of universal public health initiatives stands as the foundation for her examination and exploration of children's experiences and caregiving amid the Zambian TB and HIV crisis. Children, she writes, have largely been absent from public health initiatives of infectious disease and medical research (p. 12). In part, this erasure is rooted in Eurocentric assumptions of children as non-agentic beings, and inadequate conceptualization of children as family members (p. 15). This erasure is further compounded for child caregivers as predominant Euro-American models of childhood enacted by public health initiatives paint the caregiving and care-work done by children as child abuse. By focusing this ethnographic account on children, their voices, and experiences, Hunleth begins to rectify this erasure by critically repositioning children as social actors. She not only reminds engaged scholars that children are also impacted by the structural inequities of George and infectious disease, but also that children too "tailor global health, humanitarian, and biomedical systems of knowledge and practice to their particular circumstances" (p. 4).

A critical lens through which to consider the agency of George children is the care work and caregiving they perform for their ill parents and family members. The recent consideration of children's caregiving by social scientists has primarily focused on domestic tasks. Hunleth problematizes this "care equals work" model by focusing on the experiences of children themselves. While the children in George do variously clean house, fetch water, and complete domestic tasks, Hunleth tells us that reducing care to domestic tasks fails to account for the nuanced and affective nature of care. What is critical for both children and adults in George is the care that these children provide by staying close (geographically, residentially) to their parents, by reminding ill family members to take their medicine, and by avoiding direct naming of both TB and HIV. In extending her understanding of care beyond domestic task models, Hunleth (re)introduces the affective nature of parent-child relationships into our understanding of care. Care, in this model, is focused on maintaining the intergenerational relationships which provide both resources and safety in a constantly fluctuating context.

It becomes clear then, that childhood and children in George are inherently relational. 
Beyond the obvious cultural constructions of "child" in opposition to "adult," children and adults in George mutually rely upon each other to navigate the poverty, illness, and structural violence that characterizes their lives. As Hunleth writes,

"The resources and opportunities children [in George] are afforded depend on relationships, and children make day-to-day life meaningful and possible through their affective and practical actions. That is, interdependence characterized the relationship between adults and children" (p. 48).

It is necessary to understand then that children in George do not care for their ill parents simply because they are structurally or culturally expected to do so. Rather, engaged scholars can learn from Hunleth's work that these children receive valuable benefits by caring for their parents, including parental love, support, and material resources. With these lessons in mind, it is essential to actively incorporate acknowledgement of children's care work into biomedical treatment protocols for infectious disease.

Children as Caregivers also serves as an example of how to practically incorporate children as participants in ethnographic research. Euro-American constructions of children place less weight or value on the opinions, thoughts, and interpretations of children than those of adults. Hunleth, however, prefaces the voices of child participants. Throughout the book, she draws on workshops, role-play, and games played with the children. She incorporates children's voices into the conversation through their drawings and stories, as well as transcripts of audio-recordings the children made themselves. Relating the children's understandings of illness, she does not "correct" their interpretations, but instead acknowledges the value of their authoritative knowledge (p. 103). In so doing, Hunleth not only recognizes children as experts of their own experiences but positions children's agency at the heart of her analysis. Her inclusion of children as participants (and, critically, the children's own experiences and voices) highlights the need for engaged scholars to incorporate children as participants when research thoughtfully calls for their perspectives. We must acknowledge that children play a role equitable to adults in informing, influencing, and engaging with the economic, social, and political structures of daily life.

As a doctoral student studying the intersections of assisted reproduction and reproductive decision making, I was particularly drawn to Hunleth's central positioning of children's care work. Anthropological study of assisted reproduction and infertility has frequently considered the experiences of infertile couples, without acknowledgment of either the caregiving of/ from the other member of the couple, or any children present within the home or extended family. In Children as Caregivers, I found a reconsideration of the affective nature of familial connection, as well as an analytical focus on the interdependence central to kin relations. This has inspired me to begin to consider how children's voices, agency, and care must be centrally considered within reproductive and kinship studies.

\section{Kelsey Marr}

Ph.D. student, Interdisciplinary Graduate Studies

University of British Columbia Okanagan

Email: kelsey.marr@ubc.ca 